\section{Castleman Disease: A Rare Mimicker of Retroperitoneal Malignancy}

\section{Sir,}

The retroperitoneum can host a wide spectrum of pathologies, including a variety of rare benign and malignant neoplasms that can be either primary or metastatic in origin. ${ }^{1,2}$ Castleman's disease (CD) is a benign lymphoproliferative disorder with an incidence rate of 21 to 25 cases per million person-years. ${ }^{3,4}$ In a review of 195 CD cases located within the abdominal cavity, $63 \%$ were localised in the retroperitoneum. ${ }^{5}$

A 49-year female was referred to our clinic after detection of a mass in the retroperitoneal area as a result of examination performed in the health centre, where she presented with a 3-month history of intermittent abdominal pain. In the abdominal computerised tomography $(\mathrm{CT})$, a solid mass $(10 \times 8 \times 6 \mathrm{~cm})$ was found in the retroperitoneal area below the left renal hilum (Figure 1A). Moreover, there were millimeter-sized lymph nodes in the para-aortic area; and on PET/CT imaging, increased 18F FDG uptake was detected in the left-sided mass lesion adjacent to the psoas muscle (Figure 1B). The mass was excised by laparotomy approach (Figure 1C). On microscopic evaluation, the mass was diagnosed as hyaline vascular type of Castleman's disease (CD) (Figure 1D). The patient was followed up by the hematology clinic at 6-month intervals.

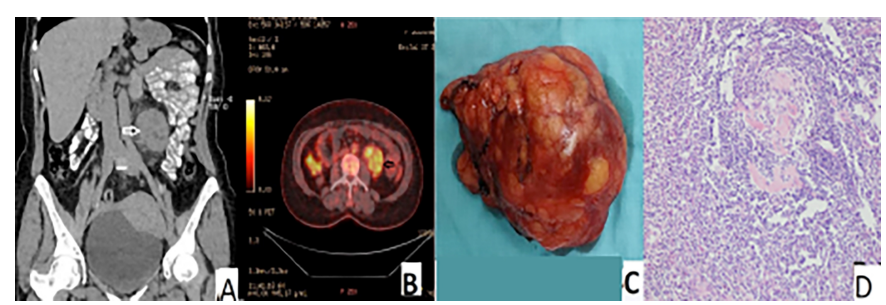

Figure 1: (A) Abdominal CT, coronal section, shows a well circumscribed and oval-shaped lesion just beneath the left kidney and adjacent to the aorta, (Arrows). (B) Abdominal PET/CT shows the lesion just beneath the left kidney and adjacent to the aorta (Arrows). (C) The gross specimen. (D) Castleman's disease of hyaline vascular type (HE, ×200).

Preoperative diagnosis of CD is difficult as there are no specific symptoms or radiographical features. Diagnostic imaging procedures that should be performed in the diagnosis of this disease include CT, MRI and PET/CT. In this patient, PET/CT was used to evaluate the differential diagnosis, localisation, and the presence of other lesions; and abdominal CT was used to evaluate the localisation and operability of the lesion. The final diagnosis is usually made after surgical removal of thetumor. ${ }^{2,4}$

If total tumor resection is impossible, partial removal or radiotherapy may help prevent the inflammatory process from becoming multicentric. Patients with multiple organ involvement should be disqualified from surgical treatment and considered for a therapy that combines glucocorticosteroids, chemotherapy and biological treatment. ${ }^{1}$
Due to the risk of lymphomatous conversion of the lymphoid masses and to the possibility of developing lymphoma in other sites, post-surgical clinical-radiological surveillance is recommended. The follow-up of these patients should, therefore, include an annual 18FFDG-PET/CT and serum biomarkers (IL-6, serum free light chains, and quantitative immunoglobulins). ${ }^{1,2}$

In conclusion, CD should be considered in the differential diagnosis of masses in the retroperitoneal location. Surgical resection of CD is still the mosteffective approach.

\section{CONFLICT OF INTEREST:}

The authors declared no conflict of interest.

\section{AUTHORS' CONTRIBUTION:}

All authors declared that they have participated in the design, execution and analysis of the paper and approved the final version to be published.

\section{REFERENCES}

1. Madan R, Chen JH, Trotman-Dickenson B, Jacobson F, Hunsaker A. The spectrum of Castleman's disease: Mimics, radiologic pathologic correlation and role of imaging in patient management. Eur J Radiol 2012; 81(1):123-31. doi: 10.1016/ j.ejrad.2010.06.018.

2. Haap M, Wiefels J, Horger M, Hoyer A, Müssig K. Clinical, laboratory and imaging findings in Castleman's disease - The subtype decides. Blood Rev 2018; 32(3):225-34. doi: 10. 1016/j.blre.2017.11.005.

3. Dispenzieri A, Fajgenbaum DC. Overview of castleman disease. Blood 2020; 135(16):1353-64. doi: 10.1182/blood. 20190 00931.

4. Sato A. Castleman's disease in the pelvic retroperitoneum: A case report and review of the Japanese literature. Int J Surg Case Rep 2013; 4(1):19-22. doi: 10.1016/j.ijscr.2012. 08.016.

5. Bucher P, Chassot G, Zufferey G, Ris F, Huber O, Morel P. Surgical management of abdominal and retroperitoneal Castleman's disease. World J Surg Oncol 2015; 3:33. doi: 10. 1186/1477-7819-3-33.

Arif Atay ${ }^{1}$, Furkan Karahan ${ }^{1}$, Sadi Bener ${ }^{2}$, Bulent Turgut ${ }^{3}$, Fatma Husniye Dilek ${ }^{2}$ and Osman Nuri Dilek ${ }^{1}$

${ }^{1}$ Department of General Surgery, Izmir Katip Celebi University Atatürk Education And Research Hospital, Izmir, Turkey ${ }^{2}$ Departman of Pathology, Izmir Katip Celebi University Atatürk Education and Research Hospital, Izmir, Turkey

${ }^{3}$ Departman of Nuclear Medicine, Izmir Katip Celebi University Atatürk Education and Research Hospital, Izmir, Turkey

Correspondence to: Dr. Arif Atay, Department of General Surgery, Izmir Katip Celebi University Atatürk Education And Research Hospital, Izmir, Turkey

E-mail: atayarif@hotmail.com

Received: July 15, 2021; Revised: March 21, 2021;

Accepted: April 22, 2021

DOI: https://doi.org/10.29271/jcpsp.2021.10.1252 